\title{
Functional Genetic Variation of the Cannabinoid Receptor I and Cannabis Use Interact on Prefrontal Connectivity and Related Working Memory Behavior
}

\author{
Marco Colizzi',2, Leonardo Fazio', Laura Ferranti' ${ }^{1,3}$, Annamaria Porcelli', Rita Masellis', Daniela Marvulli', \\ Aurora Bonvino', Gianluca Ursini ${ }^{1,4}$, Giuseppe Blasi ${ }^{1}$ and Alessandro Bertolino*, I,5 \\ 'Group of Psychiatric Neuroscience, Department of Basic Medical Sciences, Neuroscience and Sense Organs, University of Bari 'Aldo Moro', Bari, \\ Italy; '2Department of Psychosis Studies, Institute of Psychiatry, King's College London, London, UK; ${ }^{2}$ Psychiatric, Clinical Psychology and Psychiatric \\ Rehabilitation Unit, Department of General and Experimental Medicine, University of Perugia, Perugia, Italy; ${ }^{4}$ Lieber Institute for Brain \\ Development, Johns Hopkins Medical Campus, Baltimore, MD, USA; ${ }^{5}$ RRED, NORD DTA, F. Hoffman-La Roche Ltd, Basel, Switzerland
}

\begin{abstract}
Cannabinoid signaling is involved in different brain functions and it is mediated by the cannabinoid receptor I (CNRI), which is encoded by the CNRI gene. Previous evidence suggests an association between cognition and cannabis use. The logical interaction between genetically determined cannabinoid signaling and cannabis use has not been determined. Therefore, we investigated whether CNR I variation predicts CNRI prefrontal mRNA expression in postmortem prefrontal human tissue. Then, we studied whether functional variation in CNRI and cannabis exposure interact in modulating prefrontal function and related behavior during working memory processing. Thus, 208 healthy subjects ( 113 males) were genotyped for the relevant functional SNP and were evaluated for cannabis use by the Cannabis Experience Questionnaire. All individuals performed the 2-back working memory task during functional magnetic resonance imaging. CNRI rs 406977 was associated with prefrontal mRNA and individuals carrying a G allele had reduced CNRI prefrontal mRNA levels compared with AA subjects. Moreover, functional connectivity MRI demonstrated that G carriers who were also cannabis users had greater functional connectivity in the left ventrolateral prefrontal cortex and reduced working memory behavioral accuracy during the 2-back task compared with the other groups. Overall, our results indicate that the deleterious effects of cannabis use are more evident on a specific genetic background related to its receptor expression.

Neuropsychopharmacology (20I5) 40, 640-649; doi:I 0.I038/npp.20 I4.2I3; published online 24 September 20I4
\end{abstract}

\section{INTRODUCTION}

The effects of exogenous cannabis on physiology of the central nervous system are mainly modulated by cannabinoid receptor 1 (CNR1; Pertwee, 2008). This receptor is expressed in high concentrations on axons and terminals of both glial elements and neurons (Matsuda, 1997) throughout the central nervous system, including the prefrontal cortex (PFC; Pazos et al, 2005). Cannabinoid signaling is involved in various brain functions such as motor control, emotional responses, motivated behavior (Mouslech and Valla, 2009), and cognitive regulation (Ruiz-Contreras et al, 2013, 2014). It is now well established that delta-9tetrahydrocannabinol (the active ingredient of cannabis) is a CNR1 partial agonist. Like any other partial agonist,

\footnotetext{
* Correspondence: Professor A Bertolino, Department of Basic Medical Sciences, Neuroscience and Sense Organs, University of Bari, Piazza Giulio Cesare, II, BA 70124, Italy, Tel: +39 0805478572, Fax: +390805593204, E-mail: alessandro.bertolino@uniba.it

Received 2 I March 20 I4; revised 29 June 20 I4; accepted I 4 July 20 I4; accepted article preview online 19 August 2014
}

delta-9-tetrahydrocannabinol can serve both to activate or to block CNR1 receptors, depending on the expression level and coupling efficiency of these receptors (Pertwee, 2008). Consistently, several cognitive processes can be affected by delta-9-tetrahydrocannabinol (D'Souza et al, 2004; Lane et al, 2005), possibly by increasing CNR1-mediated release of acetylcholine, glutamate, and dopamine in PFC (Pertwee, 2008). However, the relationship between cannabis use and cognitive processing is still a matter of contention. Intuitively, one may expect cannabis to have a deleterious effect on cognitive performance. However, studies in healthy subjects have reported conflicting results. For example, some studies indicate that cannabis use is associated with poorer processing during cognitive functions (Bolla et al, 2002; Jockers-Scherübl et al, 2007) including working memory (Solowij et al, 2002), whereas others report no difference (Pope et al, 2001). Similar discrepancies are also present in studies in patients with schizophrenia. Some studies have reported impaired cognitive performance in patients using cannabis (Mata et al, 2008), others improved performance (Jockers-Scherübl et al, 2007; Schnell et al, 2009), and others no effect (Sevy et al, 2007). 
Along with several other factors, these discrepancies may also be because of genetic variation having an impact on these cognitive phenotypes (Barnes et al, 2011). In fact, two recent studies have reported an association between CNR1 genetic variation and working memory processing. In particular, an adenine-adenine-thymine trinucleotide repeats polymorphism and the rs2180619 single nucleotide polymorphism (SNP) of the CNR1 gene (6q14-q15) have been associated with working memory performance in healthy subjects (Ruiz-Contreras et al, 2013, 2014). However, the functional role of these polymorphisms has not been empirically demonstrated (Ruiz-Contreras et al, 2013, 2014).

The aim of this study was twofold: first, to identify functional genetic variation within CNR1 predicting mRNA levels in human postmortem PFC; second, to test whether such variation interacts with cannabis use to predict complex prefrontal phenotypes during working memory processing in psychiatrically healthy individuals. Given the relevance of cannabinoid signaling for working memory (Ruiz-Contreras et al, 2013, 2014), we hypothesized that genetic variation predicting reduced levels of prefrontal CNR1 would also interact with cannabis use to predict abnormal prefrontal activity, connectivity, and related behavior during working memory.

\section{MATERIALS AND METHODS}

CNR1 mRNA Expression in Postmortem PFC of Healthy Humans

A publicly available collection of 105 postmortem human brains of non-psychiatric Caucasian individuals with available information on sex (31 females), age at death (mean age $\pm \mathrm{SD}, 30.4 \pm 21.3$ years), $\mathrm{pH}$ of the cerebellar tissue (6.5 \pm 0.3$)$, postmortem interval in hours $(27.2 \pm 15.0)$ and RNA integrity number $(8.1 \pm 0.9)$ was used for this study. Fetal brains of this collection were excluded from the analysis because of their limited sample size (http:// braincloud.jhmi.edu/). Details of informed consent, ethics, autopsy, tissue acquisition, handling processing, dissection, clinical characterization, diagnoses, neuropathological examinations, toxicological analysis, RNA extraction, quality control measures, and DNA evaluation from cerebellar tissue were described previously (Colantuoni et al, 2011; Blasi et al, 2013a; Blasi et al, 2013b).

Sixty-one CNR1 SNPs had been typed in the Braincloud sample. Given the low number of subjects homozygous for the minor alleles, we collapsed these individuals (when present) and heterozygous subjects within one group for further analyses. This procedure is consistent with a series of earlier studies evaluating polymorphisms with low minor allele frequencies, especially when codominance of the alleles is not known (Blasi et al, 2013a). For each CNR1 SNP, postmortem prefrontal mRNA expression values were used as the dependent variable in an analysis of variance (ANOVA), with the selected SNP as the independent variable. Any statistically significant association with prefrontal mRNA expression was then controlled for the effects of covariates of no interest (age at death; postmortem interval in hours; $\mathrm{pH}$; RNA integrity number; sex) in an analysis of covariance (ANCOVA). Results were considered as significant at $p<0.05$, Bonferroni corrected for the number of CNR1 SNPs characterized in Braincloud $(N=61)$. Based on these analyses, the SNP rs 1406977 resulted significantly associated with prefrontal mRNA expression (see below, results section), and was thus considered for the in vivo analyses.

\section{CNR1 Genetic Variation and Working Memory Processing}

A total of 208 healthy subjects (113 males; mean age \pm SD, $27.50 \pm 7.79$ years) entered the study. All participants were unrelated Caucasian from the region of Puglia in Italy. Protocols and procedures were approved by the local Institutional Review Board. After complete description of the study to the subjects, written informed consent was obtained. Subjects underwent the Structured Clinical Interview for $D S M-I V$ to exclude any Axis I psychiatric disorder. Exclusion criteria were an active cannabis use in the past 6 months and during the study protocol, use of other stimulant drugs, head trauma with loss of consciousness, and any significant medical illness. Parental socioeconomical status (Hollingshead Scale, $44.14 \pm 16.52$ ), handedness (Edinburgh Inventory, $0.81 \pm 0.34$ ), and total IQ (WAIS-R, $107.64 \pm 11.33)$ were also measured.

\section{CNR1 rs1406977 Genotyping for the In Vivo Studies}

DNA was extracted from whole blood using standard procedures. Determination of CNR1 rs1406977 genotype was conducted using the $5^{\prime}$ exonuclease TaqMan assay. ANOVAs and $\chi^{2}$ were used to evaluate the relation between genotype and demographics data.

\section{Alcohol and Tobacco Use}

Participants were asked about their use of alcohol and tobacco. Those who reported alcohol use were interviewed using the Tolerance, Worried, Eye-opener, Amnesia, and Cut down (TWEAK) alcohol screening test (Chan et al, 1993). This five-question questionnaire is useful to screen for harmful drinking habits in the general population. Similarly, participants who reported tobacco use were interviewed using the Fagerström Test for Nicotine Dependence (FTND; Heatherton et al, 1991). This six-question questionnaire is useful to screen for nicotine dependence in the general population. To test the distribution of these possible confounders in our sample, $\chi^{2}$ was used to evaluate the relationship between lifetime harmful alcohol use (yes/no) and genotype/cannabis use as well as between lifetime nicotine dependence (yes/no) and genotype/cannabis use. Questionnaires were not available for the entire sample, but only for 153 subjects $(73.6 \%)$.

\section{Cannabis Use}

All participants were asked about their use of illicit drugs and those who reported cannabis use were interviewed using the Cannabis Experience Questionnaire (CEQ; Di Forti et al, 2012). This questionnaire allows the detailed assessment of lifetime patterns of cannabis and stimulants use, including age at first use, frequency and duration of use, 
and the specific type of cannabis used. ANOVAs and $\chi^{2}$ were used to evaluate the relationship between lifetime cannabis use (yes/no) and demographics data.

\section{Working Memory Task and Analysis of Behavioral Data}

All subjects completed the 2-back working memory task (Bertolino et al, 2010) during functional magnetic resonance imaging (fMRI). Briefly, $n$-back refers to how far back in the sequence of stimuli the subject has to recall. The stimuli consisted of numbers (1-4) shown in random sequence and displayed at the points of a diamond-shaped box. There was a visually paced motor task that also served as a nonmemory-guided control condition (0-back) that simply required subjects to identify the stimulus currently seen. In the working memory condition, the task required recollection of a stimulus seen two stimuli previously (2-back) while continuing to encode additionally incoming stimuli.

A factorial ANOVA, with cannabis use (yes/no) and CNR1 rs1406977 genotype (AA, G carrier) as independent variables, was used to evaluate the main effects as well as the interaction between cannabis use and rs 1406977 on working memory performance. A factorial ANOVA was also performed in the 153 individuals with available information on tobacco and alcohol use. Furthermore, to control for the potential confounding effect of nicotine and alcohol use, a factorial ANCOVA was performed by adding these variables as covariates. The statistical threshold was set at $p<0.05$. Fisher's test was used for post hoc analyses.

\section{fMRI Data Acquisition}

Blood oxygen level-dependent fMRI was performed on a GE Signa $3 \mathrm{~T}$ scanner (General Electric), equipped with a standard quadrature head coil as previously described (Bertolino et al, 2010). A gradient-echo planar imaging sequence (repetition time, $2000 \mathrm{~ms}$; echo time, $28 \mathrm{~ms} ; 20$ interleaved axial slices; thickness, $4 \mathrm{~mm}$; gap, $1 \mathrm{~mm}$; voxel size, $3.75 \mathrm{~mm}$ (isotropic); flip angle, $90^{\circ}$; field of view, $24 \mathrm{~cm}$; matrix, $64 \times 64)$ was used to acquire 120 volumes while subjects performed the 2-back working memory task. The first four scans were discarded to allow for a T1 equilibration effect.

\section{fMRI Data Analysis}

Analysis of the fMRI data was completed using statistical parametric mapping (SPM8; http://www.fil.ion.ucl.ac.uk/ $\mathrm{spm})$. All preprocessing was performed as described previously (Blasi et al, 2013b). Following preprocessing, all fMRI data were analyzed using General Linear Model (GLM) to detect changes in brain activity based on a priori task design and hemodynamic response function. We also used Independent Component Analysis (ICA) to identify patterns of higher-order functional connectivity within multiple networks during the same task, each of which subserves a specific subprocess (functional covariance).

\section{General Linear Model}

The individual contrast images (2- vs 0 -back) were used in second-level random-effects models to determine task- specific regional responses at the group level. In particular, factorial ANOVA was performed on this contrast, entering cannabis use (yes/no) and CNR1 rs1406977 genotypes (AA, $\mathrm{G}$ carrier) as independent variables. A factorial ANOVA was also performed in the 153 individuals with available information on tobacco and alcohol use. Furthermore, to control for the potential confounding effects of nicotine, alcohol, and behavioral performance at the task, factorial ANCOVAs were performed by adding these variables as covariates. We used a family-wise small volume-corrected statistical threshold of $p$ value $<0.05$ (minimum cluster size $[\mathrm{k}]=5$ ), using as volume of interest the Wake Forest University PickAtlas (http://fmri.wfubmc.edu/cms/software \#PickAtlas) Brodmann's areas (BAs) of the dorsolateral PFC (DLPFC). These regions of interest were a priori chosen based on our investigation of prefrontal phenotypes and on the well-established association between the $n$-back task and activity in the dorsolateral PFC (Bertolino et al, 2010; Blasi et al, 2013b).

\section{Independent Component Analysis}

A group spatial ICA was performed using the infomax algorithm (Bell and Sejnowski, 1995) within the GIFT toolbox (http://icatb.sourceforge.net). fMRI datasets were split into a final set of 28 spatially independent components (ICs), using a modified minimum description length algorithm (Li et al, 2007). This is a stochastic process and the end results are not always identical. To address this issue, we reran ICA for 50 iterations using ICASSO (Himberg et al, 2004), an optimization algorithm that repeats ICA analysis multiple times and finds the degree to which ICs are consistent between different runs. Finally, IC timecourses and spatial maps (functional connectivity maps) were back-reconstructed for each participant (Calhoun et al, 2001). Back-reconstructed spatial maps of each IC were normalized into z-scores (Beckmann et al, 2005) that reflect the spatial map contribution to the associated timecourse. The normalized spatial maps of $z$-scores of each participant were averaged together across the three runs and entered into second-level random effect analysis (one-sample $t$ test). The $t$-maps were used to identify the brain regions involved in each IC. On the basis of previous studies that applied ICA to large samples (Schmithorst et al, 2006, 2007), the significance threshold was set at $p<1 \times 10^{-10}$ (voxel level), family-wise error whole-brain corrected, minimum cluster size $[k]=5$.

\section{Component Selection}

A systematic procedure was used to individuate Functional Networks (FNs). We used the probabilistic maps of white matter, cerebrospinal fluid, and gray matter in MNI space provided with SPM8 as templates, and Multiple Linear Regression implemented in the spatial sorting function of GIFT to compare the spatial pattern of each IC with these templates. This analysis generated three correlation coefficients $\left(r^{2}\right)$ for every IC, one for each template. Components that scored a higher spatial correlation value with cerebrospinal fluid $\left(r^{2}>0.05\right)$ and with white matter $\left(r^{2}>0.02\right)$ maps and/or a lower spatial correlation value with gray 
matter map $\left(r^{2}<0.01\right)$ were not considered to be meaningful activations in a manner similar to previous studies (Kim et al, 2009; Sambataro et al, 2010). Therefore, seven ICs (IC 1, 3, 4, 5, 7, 8, 14) were excluded from further analysis. The remaining $21 \mathrm{ICs}$ were labeled as FNs and were subjected to our second criterion of experimental task relevance. We were interested in components that were highly correlated with the experimental design, the $n$-back task. Therefore, each subject's GLM canonical hemodynamic response model from SPM8 was entered into a temporal sorting analysis using the GIFT software to compare the temporal pattern of each IC in relation to the $n$-back task. This analysis generated a correlation coefficient $\left(r^{2}\right)$ for every FN. Only eight FNs (IC $2,9,12,16,17$, $23,27,28)$ showed a positive correlation with the $n$-back task. Finally, we used the cluster quality index $\left(I_{\mathrm{q}}\right)$ to validate ICA reliability too. Components that resulted unstable as suggested by a coefficient of stability $\left(I_{\mathrm{q}}\right)$ lower than 0.95 (Himberg et al, 2004; Sambataro et al, 2010) were removed from the analyses. Five FNs $\left(0.86<I_{\mathrm{q}}<0.93\right)$ were excluded. Only three FNs showed an $I_{\mathrm{q}}>0.95$ (IC 2, 9, 17) and the $t$-maps were selected for further analyses. As described for the GLM approach, factorial ANOVAs were performed on these FNs entering cannabis use (yes/no) and CNR1 rs1406977 genotypes (AA, G carrier) as independent variables and brain connectivity during working memory as the dependent variable. Factorial ANOVAs were also performed in the 153 individuals with available information on tobacco and alcohol use. Furthermore, to control for the potential confounding effects of nicotine, alcohol, and behavioral performance at the task, factorial ANCOVAs were performed by adding these variables as covariates. We used a family-wise error-corrected statistical threshold of $p$ value $<0.05$ (minimum cluster size $[k]=5$ ). Fisher's test was used for post hoc analyses.

\section{RESULTS}

\section{Association of CNR1 SNPs with Postmortem CNR1 Prefrontal mRNA Expression}

CNR1 mRNA expression in the Braincloud sample was normally distributed. Out of the available 61 CNR1 SNPs, only rs1406977 was associated with mRNA expression in postmortem $\operatorname{PFC}(\mathrm{F}(1,104)=13.9$, corrected $p=0.019)$. Fisher's post hoc analysis revealed that $\mathrm{G}$ carriers (40 AG +2 GG individuals) have lower prefrontal CNR1 levels compared with AA subjects (63 individuals) (Figure 1). Moreover, this association was also significant when covarying the analysis for sex, RNA integrity number, postmortem interval in hours, $\mathrm{pH}$ of the cerebellar tissue, and age.

\section{In vivo Study: Allelic Distribution of the CNR1 rs1406977 Genotype and Demographic Measures}

The allelic distribution of the rs 1406977 genotype in the 208 healthy subjects included in the in vivo study was as follows: 125 AA subjects, 74 AG subjects, 9 GG subjects. As described above, AG and GG subjects were collapsed within one group. After genotype determination, groups displayed Hardy-Weinberg equilibrium $(p>0.1)$. The two genotype groups (AA, G carriers) did not differ for any demographic

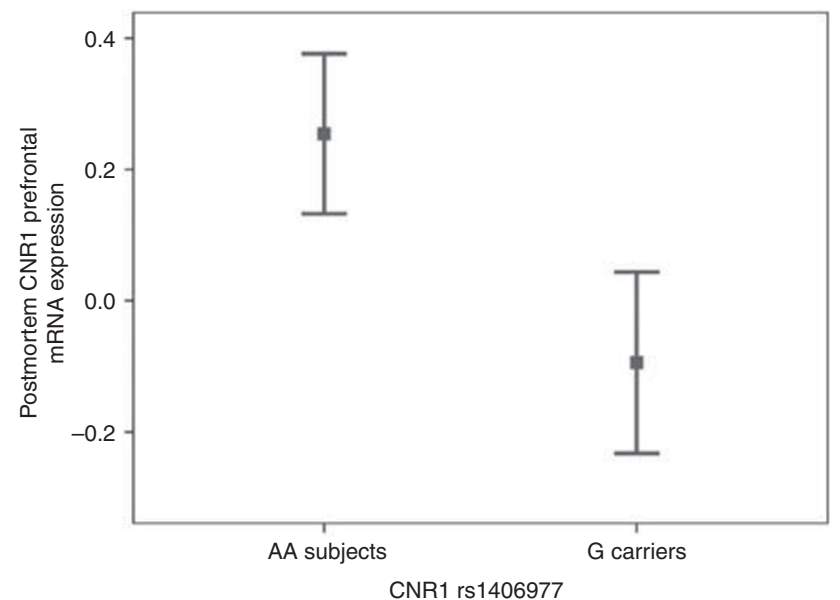

Figure I Association between CNRI rs/406977 and postmortem CNRI prefrontal mRNA expression.

measure (sex, age, parental socioeconomical status, handedness, and total IQ, all $p>0.1$ ). Similarly, there were no significant differences in demographics between cannabis users and cannabis-naïve subjects (Table 1 , all $p>0.1$ ).

\section{Patterns of Alcohol and Tobacco Use}

Data were available for 153 subjects. Seventy-three individuals (48\%) reported lifetime harmful alcohol use. The two genotype groups did not differ in their pattern of alcohol use. Similarly, there was no significant difference in their pattern of alcohol use between cannabis users and cannabisnaïve subjects (Table 1 , all $p>0.1$ ).

Sixty-five individuals $(42 \%)$ reported lifetime nicotine dependence. The two genotype groups did not differ in their pattern of tobacco use (Table $1, p>0.1$ ). Instead, cannabis users tended to be more frequently tobacco smokers with nicotine dependence (Table $1 ; \chi^{2}=3.14$, $p=0.076)$.

\section{Pattern of Cannabis Use}

Out of 208 individuals, 91 (44\%) reported lifetime cannabis use. Among cannabis users, no subject was a current cannabis user, all individuals reporting an abstinence period of at least 6 months. Pattern of cannabis use (age at first use, duration of use, frequency of use, and type used) is reported in Table 2. The two genotype groups did not differ in their pattern of cannabis use (Table 2, all $p>0.1$ ).

\section{Working Memory Behavioral Performance}

Factorial ANOVA on accuracy data demonstrated: a main effect of CNR1 rs1406977, with G carrier subjects having reduced accuracy compared with AA subjects $\left(\mathrm{F}_{1,207}=6.26\right.$; $p=0.013$ ); a main effect of cannabis use approaching significance, with cannabis users tending to have reduced accuracy compared with cannabis-naïve subjects $\left(\mathrm{F}_{1,207}=\right.$ $3.35 ; p=0.068)$; and an interaction between genotype and cannabis use $\left(\mathrm{F}_{1,204}=4.61 ; p=0.033\right)$. Fisher's post hoc analysis revealed that, although cannabis use did not 
Table I Demographic Variables and Patterns of Lifetime Alcohol and Tobacco Use

\begin{tabular}{|c|c|c|c|c|}
\hline & $\begin{array}{l}\text { CNRI rsI406977 AA subjects } \\
\qquad n=125 \text { (65 males) }\end{array}$ & $\begin{array}{l}\text { CNRI rs I } 406977 \text { G carriers } \\
\qquad n=83 \text { (45 males) }\end{array}$ & $\begin{array}{l}\text { Cannabis-naïve subjects } \\
\qquad n=1 \text { I } 7 \text { (58 males) }\end{array}$ & $\begin{array}{l}\text { Cannabis users } \\
n=91 \text { (55 males) }\end{array}$ \\
\hline Age & $27.14(6.89)$ & $27.99(8.96)$ & $28.34(8.80)$ & $26.74(6.26)$ \\
\hline PSES & $45.11(16.64)$ & $42.09(16.32)$ & $43.54(16.83)$ & $44.38(16.24)$ \\
\hline Handedness & $0.81(0.31)$ & $0.83(0.39)$ & $0.84(0.34)$ & $0.78(0.35)$ \\
\hline IQ & $n(\%)$ & $n(\%)$ & $n(\%)$ & n (\%) \\
\hline \multicolumn{5}{|l|}{ Alcohol use } \\
\hline HD yes & $48(50.5)$ & $25(43.1)$ & $34(42)$ & $39(54.2)$ \\
\hline HD no & $47(49.5)$ & $33(56.9)$ & $47(58)$ & $33(45.8)$ \\
\hline No details & 30 & 25 & 36 & 19 \\
\hline No details & 30 & 25 & 36 & 19 \\
\hline
\end{tabular}

Abbreviations: HD, harmful drinking; IQ, Intelligence Quotient; ND, nicotine dependence; PSES, parental socio-economical status.

Table 2 Pattern of Lifetime Cannabis Use

\begin{tabular}{|c|c|c|c|}
\hline & Sample & $\begin{array}{l}\text { CNRI rs I } 406977 \\
\text { AA subjects }\end{array}$ & $\begin{array}{l}\text { CNRI rsI406977 } \\
\text { G carriers }\end{array}$ \\
\hline & n (\%) & $n(\%)$ & n (\%) \\
\hline & $208(100)$ & $125(100)$ & $83(100)$ \\
\hline \multicolumn{4}{|l|}{ Ever used } \\
\hline No & $117(56.25)$ & $66(52.8)$ & $51(61.4)$ \\
\hline Yes & $91(43.75)$ & $59(47.2)$ & $32(38.6)$ \\
\hline \multicolumn{4}{|l|}{ Age at first use $\mathrm{a}^{\mathrm{a}}$} \\
\hline Under 17 years & $35(38.5)$ & $25(42.4)$ & $10(31.25)$ \\
\hline 17 years and over & $56(6 \mid .5)$ & $34(57.6)$ & $22(68.75)$ \\
\hline \multicolumn{4}{|l|}{ Duration of use $^{\mathrm{a}}$} \\
\hline $\begin{array}{l}\text { Use for less than } \\
5 \text { years }\end{array}$ & $85(93.4)$ & $55(93.2)$ & $30(93.75)$ \\
\hline $\begin{array}{l}\text { Use for more than } \\
5 \text { years }\end{array}$ & $6(6.6)$ & $4(6.8)$ & $2(6.25)$ \\
\hline \multicolumn{4}{|l|}{ Frequency of use $\mathrm{e}^{\mathrm{a}}$} \\
\hline Occasional use & $48(52.7)$ & $36(6 \mathrm{I})$ & $12(37.5)$ \\
\hline Monthly use & $19(20.9)$ & II (18.6) & $8(25)$ \\
\hline Weekly use & $14(15.4)$ & $8(13.6)$ & $6(18.75)$ \\
\hline Daily use & $10(11)$ & $4(6.8)$ & $6(18.75)$ \\
\hline \multicolumn{4}{|l|}{ Type used ${ }^{\mathrm{a}}$} \\
\hline Resin/herbal cannabis & $91(100)$ & $59(100)$ & $32(100)$ \\
\hline Sinsemilla (skunk) & - & - & - \\
\hline
\end{tabular}

aln those who had ever used cannabis.

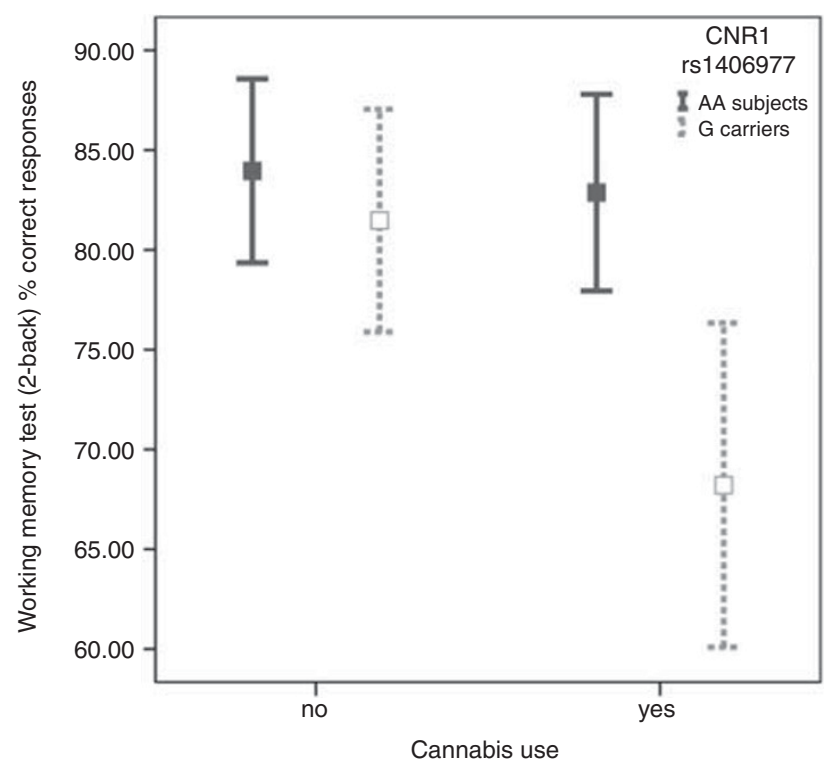

Figure 2 Interaction between CNRI rs|406977 and cannabis use on healthy subjects' working memory test (2-back) accuracy.

significantly affect working memory performance in the context of AA genotype, G-carrying cannabis users performed less accurately than G-carrying cannabis-naïve subjects $(p=0.006)$, AA cannabis users $(p=0.001)$, and AA cannabis-naïve subjects $(p<0.001)$ (Figure 2$)$.

ANOVA on reaction time data revealed a main effect of CNR1 rs1406977, with G carriers subjects having greater reaction time than AA individuals $\left(\mathrm{F}_{1,207}=3.93 ; p=0.049\right)$. Moreover, there was a main effect of cannabis use. Here, 
cannabis users had greater reaction time compared with cannabis-naïve subjects $\left(F_{1,207}=4.00 ; p=0.047\right)$. No significant interaction was found between genotype and cannabis use on reaction time.

ANOVA on accuracy data in the 153 subjects with available information on tobacco and alcohol use demonstrated: a main effect of CNR1 rs1406977, with G carriers having reduced accuracy compared with AA subjects $\left(\mathrm{F}_{1,152}=6.24 ; \quad p=0.014\right) ;$ and an interaction between genotype and cannabis use $\left(\mathrm{F}_{1,149}=4.98 ; p=0.027\right)$. Fisher's post hoc analysis revealed that, although cannabis use did not significantly affect working memory performance in the context of AA genotype, G-carrying cannabis users performed less accurately than G-carrying cannabis-naïve subjects $(p=0.014)$, AA cannabis users $(p<0.001)$, and AA cannabis-naïve subjects $(p=0.003)$. No significant main effect of cannabis use was found on accuracy.

ANOVA in this reduced sample of subjects did not reveal any statistically significant main effect or interaction of rs1406977 and cannabis use on reaction time.

Further ANCOVAs on accuracy and reaction time using harmful drinking behavior and nicotine dependence as covariates demonstrated behavioral findings almost identical to ANOVAs results, indicating that these potentially confounding variables do not affect the behavioral results. In particular, ANCOVA on accuracy demonstrated: a main effect of CNR1 rs1406977, with G carrier subjects having reduced accuracy compared with AA subjects $\left(\mathrm{F}_{1,152}=6.06\right.$; $p=0.015)$; and an interaction between genotype and cannabis use $\left(\mathrm{F}_{1,149}=4.96 ; p=0.027\right)$. Fisher's post hoc analysis revealed that, although cannabis use did not significantly affect working memory performance in the context of AA genotype, G-carrying cannabis users performed less accurately than G-carrying cannabis-naïve subjects $(p=0.014)$, AA cannabis users $(p<0.001)$, and AA cannabis-naïve subjects $(p=0.003)$.

\section{fMRI Results}

General linear model results of the working memory paradigm

CNR1 rs1406977 and cannabis use main effects and interaction during working memory. Factorial ANOVA did not reveal any statistically significant main effect or interaction of rs1406977 and cannabis use on brain activity at the chosen statistical threshold. Addition of the covariates describing nicotine and alcohol use or subjects' behavioral performance in the statistical model did not modify the results.

\section{Independent component analysis results of the working memory paradigm}

Component description. As previously described, three components were found as most task-related, ie IC 2, 9, and 17. Specifically, component 2 identified primarily the bilateral parietal lobe and included the superior posterior parietal cortex (BA 7). Additional involvement of the DLPFC was also present in this component (Posterior Parietal Component). Component 9 identified primarily the DLPFC bilaterally (BA 9/46) (Bilateral DLPFC Component). Component 17 identified primarily the left VLPFC (BA 44/ 47) with additional involvement of the temporal lobe (Left
VLPFC Component), in line with previous results on neural substrates of semantic/linguistic processing during verbal/ nonspatial working memory tasks (Thompson-Schill et al, 1997; Wagner et al, 2001; He et al, 2012).

CNR1 rs1406977, cannabis use, and brain connectivity during working memory. Factorial ANOVAs did not reveal any statistically significant main effect or interaction of rs1406977 and of cannabis use on the Posterior Parietal Component (IC2) or on the Bilateral DLPFC Component (IC 9). Furthermore, there was no statistically significant main effect of CNR1 rs1406977 or cannabis use on the Left VLPFC Component (IC 17) connectivity. Instead, there was a significant interaction of CNR1 rs1406977 and cannabis use on the latter component (BA 47, $x=-40, y=30$, $\left.z=-8, Z=4.18, \mathrm{k}=158, p_{\mathrm{FWE}-\mathrm{corrected}}=0.02\right)$. Post hoc analysis revealed a double dissociation. More specifically, there was greater connectivity in G-carrying cannabis users relative to G-carrying cannabis-naïve subjects $(p=0.004)$ and an inverse pattern in AA subjects $(p<0.001)$. Similarly, although in the cannabis users group $G$ carriers showed greater connectivity than AA subjects $(p=0.002)$, the opposite pattern was present in the cannabis-naive group $(p=0.002)$ (Figure 3).

Factorial ANOVAs in the 153 individuals with available information on tobacco and alcohol use did not reveal any statistically significant main effect or interaction of rs1406977 and of cannabis use on the Posterior Parietal Component (IC2) or on the Bilateral DLPFC Component (IC 9). Furthermore, factorial ANOVA did not reveal any statistically significant main effect of CNR1 rs1406977 or cannabis use on the Left VLPFC Component (IC 17) connectivity. Instead, there was an interaction of CNR1 rs1406977 with cannabis use on the Left VLPFC Component $(Z=3.52)$. Post hoc analysis revealed a double dissociation. More specifically, there was greater connectivity in G-carrying cannabis users relative to G-carrying cannabis-naïve subjects $(p<0.001)$ and an inverse pattern in AA subjects $(p=0.018)$. Similarly, although in the cannabis user group $\mathrm{G}$ carriers showed greater connectivity than AA subjects $(p<0.001)$, the opposite pattern was present in the cannabis-naïve group ( $p=0.019)$.

Further ANCOVAs adding the covariates describing nicotine and alcohol use in the statistical model demonstrated an interaction of CNR1 rs1406977 with cannabis use on the Left VLPFC Component $(Z=3.56)$, indicating that these potentially confounding variables do not significantly change the results.

Finally, ANCOVAs using subjects' behavioral performance as a covariate demonstrated an interaction of CNR1 rs1406977 with cannabis use on the Left VLPFC Component $(Z=4.29)$, indicating that this potentially confounding variable do not significantly change the results.

\section{DISCUSSION}

The present results indicate that rs1406977 is associated with differential CNR1 mRNA expression in postmortem human PFC. Moreover, this polymorphism interacts with cannabis use in modulating ventrolateral PFC (VLPFC) connectivity and behavior during working memory. These 

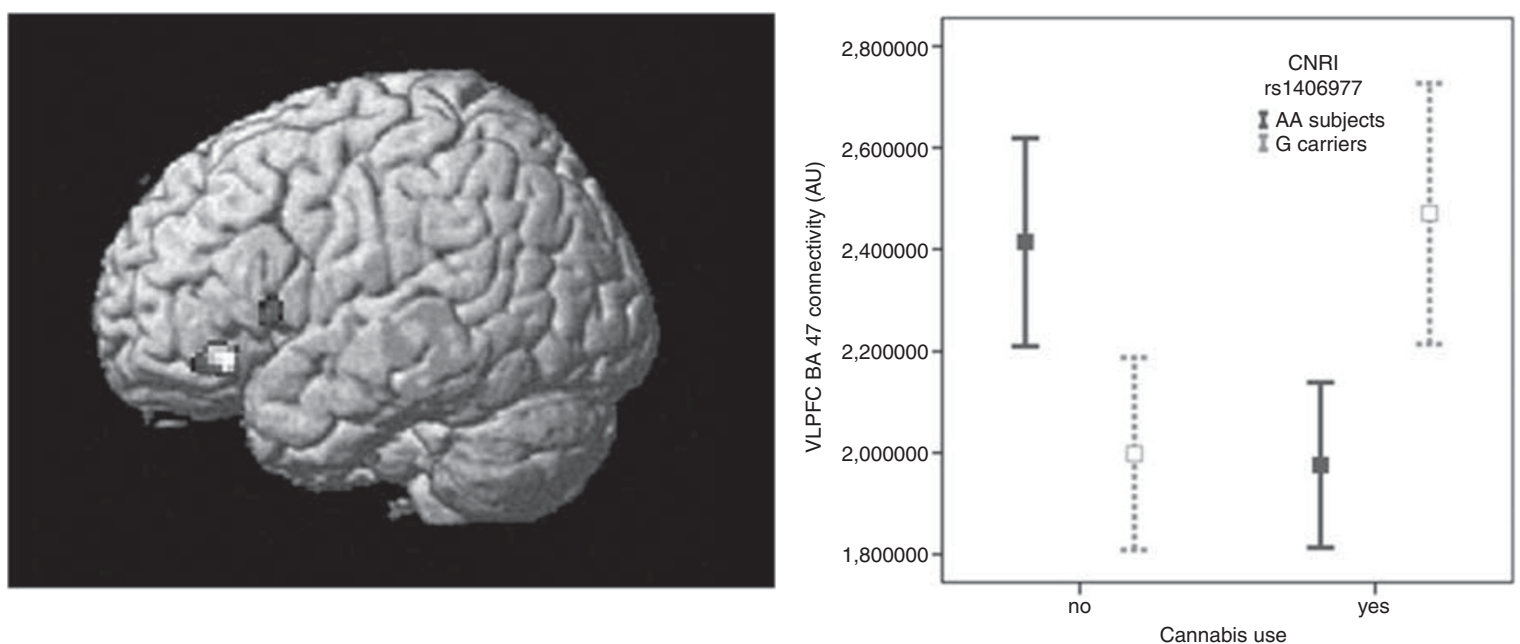

Figure 3 Interaction between CNRI rs|406977 and cannabis use on healthy subjects' Left VLPFC Component (IC I7) during Working Memory Test (2-back).

results shed new light on the potential for cannabis use to alter working memory prefrontal connectivity and related behavior based on specific genetic backgrounds. Moreover, they provide another instance of the multiple possible interactions between environmental factors and genetic variation on brain activity and behavior.

Our candidate for an association between CNR1 and working memory dysfunction was the CNR1 rs1406977 polymorphism. Our postmortem findings suggested a functional role of this SNP in modulating prefrontal CNR1 mRNA expression, with $G$ carriers having reduced prefrontal CNR1 levels compared with AA subjects. Given the potential for this association to alter physiological patterns of brain response, we investigated whether rs1406977 and cannabis use together concur in modulating complex phenotypes at greater biological distance from gene effects and including brain connectivity during working memory and related behavior. At the behavioral level, we find an interaction between cannabis use and rs 1406977 on working memory behavioral performance in healthy subjects. In particular, in the context of cannabis use, rs1406977 G carriers, who had significantly lower CNR1 mRNA levels than AA subjects, have reduced accuracy at the 2-back task compared with the other groups. Instead, cannabis use did not significantly affect working memory performance in the context of AA genotype. This result extends previous reports on association of genetic variability in CNR1 (adenineadenine-thymine trinucleotide repeat and rs2180619 polymorphisms) with cognitive behavior (Ruiz-Contreras et al, $2013,2014)$. To understand the functional significance of rs2180619, we evaluated the potential association of rs9353528 (which is in high linkage disequilibrium $r^{2}=0.934, \mathrm{D}^{\prime}=1.0$ ) with mRNA expression of CNR1 in Braincloud. However, this SNP is not associated with expression (results not shown, $\mathrm{F}_{2,104}=0.28$; uncorrected $\left.p=0.75\right)$ indicating that this is not the mechanism explaining the earlier association (RuizContreras et al, 2014). Overall, our result suggests a moderating effect of variation in CNR1 on the relationship between cannabis use and cognition, and may implicate the $G$ allele of rs1406977 in genetic vulnerability to cannabis-induced altered cognition. Consistently, other studies have indicated that variation in specific genes may influence the risk for cannabis users to develop altered cognition (van Winkel et al, 2011a; Verdejo-García et al, 2013).

Specific brain imaging phenotypes in our study were also affected by genetic variation in CNR1 and cannabis use. In fact, their interaction has a significant impact on prefrontal connectivity as measured with ICA. More in detail, we find that G-carrying cannabis users, who have poorer behavioral performance, also have greater VLPFC connectivity during the $n$-back task. In line with well-established models of prefrontal function (Gottesman and Gould, 2003), this result may be interpreted as the need of G-carrying cannabis users for greater prefrontal connectivity to perform a working memory task with lower levels of proficiency. In other words, the interaction between the G allele of rs 1406977 and cannabis use elicits inefficient prefrontal connectivity during working memory.

Interestingly, we find a gene by cannabis use interaction on the VLPFC ICA component only. VLPFC has been reported to play a critical role in guiding the selection and retrieval of semantic/linguistic knowledge (non spatial data) (Thompson-Schill et al, 1997; Wagner et al, 2001). Furthermore, previous studies have consistently indicated that cannabinoids exert a profound influence on synaptic plasticity underlying learning and memory. Verbal learning and memory have been perhaps the most consistently impaired cognitive functions in studies of acute cannabis administration and in long-term cannabis users (Solowij and Michie, 2007), as well as in heavy or long-term cannabis users in the unintoxicated state (Pope et al, 2001; Solowij et al, 2002; Grant et al, 2003). Thus, these previous findings and the present results suggest that genetically determined lower prefrontal CNR1 levels are associated with abnormalities in the neuronal networks related with verbal working memory in healthy cannabis users.

On the other hand, based on GLM analysis, we do not find an interaction between rs1406977 and cannabis use on prefrontal activity during working memory. Divergent findings on prefrontal activity and connectivity in our study may be related in part by the fact that GLM and ICA approaches likely investigate different physiological 
mechanisms (Xu et al, 2013). More in detail, GLM analysis detects changes in brain activity based on a priori task design and hemodynamic response function (functional specificity). Instead, ICA is a multivariate statistical approach that detects patterns of functional connectivity within multiple networks, identifying multiple spatially independent and temporally synchronous activity patterns in brain regions (functional covariance) (Xu et al, 2013). Thus, it is possible that the VLPFC functional connectivity better identifies the interaction between CNR1 genotype and cannabis use compared with localized activity in prefrontal areas. Moreover, task performance might have masked genotype association with brain functional activity during working memory. However, when including subjects' performance as covariate, the results were not significantly affected.

A previous report (Kanayama et al, 2004) indicated greater PFC activity during working memory in spite of similar behavioral performance in cannabis users compared with non-users. In the present study, we do not find a main effect of cannabis use on working-memory-related prefrontal activity. Lack of consistency between our results and this previous study can be in part be related to characteristics of the samples investigated. For example, we studied long abstinent (at least 6 months of abstinence) individuals with prevalent slight/mild pattern of cannabis use. Differently, this previous study examined brain activity in a population of long-term heavy cannabis users after $6-36 \mathrm{~h}$ from the last cannabis use.

A previous study reported a CNR1 rs2023239 by cannabis use interaction on bilateral hippocampus volumes (Schacht et al, 2012). Because this SNP has been associated with differential CNR1 mRNA expression in PFC (Hutchison et al, 2008), our study together with this previous report suggest that CNR1 variation predicting differential prefrontal receptor expression can also predict alteration in memory-related brain structures and functions in the context of cannabis use. Therefore, depending on the expression level of CNR1 receptors, some individuals seem to be more vulnerable to the cannabis-induced deleterious effects on regional specific brain structures and functions associated with memory processing.

The presence of only a limited number of subjects with a pattern of heavy cannabis use in our sample (daily use and/ or use for more than 5 years) is a limitation that needs to be taken into account. Although we used 'lifetime cannabis use' in the gene by cannabis interaction, we cannot further explore 'frequency of use' because of the very limited statistical power. Moreover, although including only Caucasians was useful to avoid any potential effect of population stratification, this selection limited the generalizability of our results to other ethnicities.

Working memory deficits and prefrontal anomalies are crucially associated with schizophrenia (Weinberger et al, 2001). Furthermore, convergent findings suggest that CNR1 signaling may be abnormal in patients with this brain disorder, even if the functional specificity of these anomalies is unclear (Dalton et al, 2011). Moreover, CNR1 has been inconsistently associated with diagnoses of schizophrenia (Chavarría-Siles et al, 2008; Seifert et al, 2007), and genetic variability and cannabis use appear to interact in conferring risk for psychosis (Caspi et al, 2005; van Winkel et al, 2011b, Di Forti et al, 2012). Interestingly, CNR1 signaling has been implicated in dopamine signaling (Bloomfield et al, 2014), which is centrally linked with schizophrenia (Weinberger et al, 2001). Therefore, further investigation of the putative relationship between the present findings and schizophrenia is warranted.

\section{FUNDING AND DISCLOSURE}

The authors declare no conflict of interest.

\section{ACKNOWLEDGEMENTS}

We would like to thank Dr Paolo Taurisano, Dr Raffaella Romano, Dr Barbara Gelao, Dr Marina Mancini, Dr Tiziana Quarto, Dr Francesca Ferrante, Dr Giovanna Todarello, Dr Giuseppe Rizzo, and Riccarda Lomuscio for help with data acquisition, Dr Rosalia Costa for helpful discussions, and all the people who voluntarily took part to this study. In this study, there is no funding source. Prof Alessandro Bertolino is a full-time employee of Hoffman-La Roche Ltd.

\section{REFERENCES}

Barnes JJ, Dean AJ, Nandam LS, O’Connell RG, Bellgrove MA (2011). The molecular genetics of executive function: role of monoamine system genes. Biol Psychiatry 69: 127-143.

Beckmann CF, DeLuca M, Devlin JT, Smith SM (2005). Investigations into resting-state connectivity using independent component analysis. Philos Trans R Soc Lond B Biol Sci 360: 1001-1013.

Bell AJ, Sejnowski TJ (1995). An information-maximization approach to blind separation and blind deconvolution. Neural Comput 7: 1129-1159.

Bertolino A, Taurisano P, Pisciotta NM, Blasi G, Fazio L, Romano R et al (2010). Genetically determined measures of striatal D2 signaling predict prefrontal activity during working memory performance. PLoS One 5: e9348.

Blasi G, De Virgilio C, Papazacharias A, Taurisano P, Gelao B, Fazio L et al (2013a). Converging evidence for the association of functional genetic variation in the serotonin receptor $2 \mathrm{a}$ gene with prefrontal function and olanzapine treatment. JAMA Psychiatry 70: 921-930.

Blasi G, Napolitano F, Ursini G, Di Giorgio A, Caforio G, Taurisano P et al (2013b). Association of GSK-3 $\beta$ genetic variation with GSK$3 \beta$ expression, prefrontal cortical thickness, prefrontal physiology, and schizophrenia. Am J Psychiatry 170: 868-876.

Bloomfield MA, Morgan CJ, Egerton A, Kapur S, Curran HV, Howes OD (2014). Dopaminergic function in cannabis users and its relationship to cannabis-induced psychotic symptoms. Biol Psychiatry 75: 470-478.

Bolla KI, Brown K, Eldreth D, Tate K, Cadet JL (2002). Dose-related neurocognitive effects of marijuana use. Neurology 59: 1337-1343.

Calhoun VD, Adali T, Pearlson GD, Pekar JJ (2001). A method for making group inferences from functional MRI data using independent component analysis. Hum Brain Mapp 14: 140-151.

Caspi A, Moffitt TE, Cannon M, McClay J, Murray R, Harrington H et al (2005). Moderation of the effect of adolescent-onset cannabis use on adult psychosis by a functional polymorphism in the catechol-O-methyltransferase gene: longitudinal evidence of a gene $\mathrm{X}$ environment interaction. Biol Psychiatry 57: $1117-1127$.

Chan AW, Pristach EA, Welte JW, Russell M (1993). Use of the TWEAK test in screening for alcoholism/heavy drinking in three populations. Alcohol Clin Exp Res 17: 1188-1192. 
Chavarría-Siles I, Contreras-Rojas J, Hare E, Walss-Bass C, Quezada P, Dassori A et al (2008). Cannabinoid receptor 1 gene (CNR1) and susceptibility to a quantitative phenotype for hebephrenic schizophrenia. Am J Med Genet B Neuropsychiatr Genet 147: 279-284.

Colantuoni C, Lipska BK, Ye T, Hyde TM, Tao R, Leek JT et al (2011). Temporal dynamics and genetic control of transcription in the human prefrontal cortex. Nature 478: 519-523.

D’Souza DC, Perry E, MacDougall L, Ammerman Y, Cooper T, Wu YT et al (2004). The psychotomimetic effects of intravenous delta-9-tetrahydrocannabinol in healthy individuals: implications for psychosis. Neuropsychopharmacology 29: 1558-1572.

Dalton VS, Long LE, Weickert CS, Zavitsanou K (2011). Paranoid schizophrenia is characterized by increased CB1 receptor binding in the dorsolateral prefrontal cortex. Neuropsychopharmacology 36: 1620-1630.

Di Forti M, Iyegbe C, Sallis H, Kolliakou A, Falcone MA, Paparelli A et al (2012). Confirmation that the AKT1 (rs2494732) genotype influences the risk of psychosis in cannabis users. Biol Psychiatry 72: 811-816.

Heatherton TF, Kozlowski LT, Frecker RC, Fagerström KO (1991). The Fagerström Test fot Nicotine Dependence: a revision of the Fagerström Tolerance Questionnaire. $\mathrm{Br} J$ Addict 86: $1119-1127$.

Hutchison KE, Haughey H, Niculescu M, Schacht J, Kaiser A, Stitzel J et al (2008). The incentive salience of alcohol: translating the effects of genetic variant in CNR1. Arch Gen Psychiatry 65: 841-850.

Gottesman II, Gould TD (2003). The endophenotype concept in psychiatry: etymology and strategic intentions. Am J Psychiatry 160: 636-645.

Grant I, Gonzalez R, Carey CL, Natarajan L, Wolfson T (2003). Non-acute (residual) neurocognitive effects of cannabis use: a meta-analytic study. Int Neuropsychol Soc 9: 679-689.

He H, Sui J, Yu Q, Turner JA, Ho BC, Sponheim SR et al (2012). Altered small-world brain networks in schizophrenia patients during working memory performance. PLoS One 7: e38195.

Himberg J, Hyvärinen A, Esposito F (2004). Validating the independent components of neuroimaging time series via clustering and visualization. Neuroimage 22: 1214-1222.

Jockers-Scherübl MC, Wolf T, Radzei N, Schlattmann P, Rentzsch J, Gómez-Carrillo de Castro A et al (2007). Cannabis induces different cognitive changes in schizophrenic patients and in healthy controls. Prog Neuropsychopharmacol Biol Psychiatry 31: 1054-1063.

Kanayama G, Rogowska J, Pope HG, Gruber SA, Yurgelun-Todd DA (2004). Spatial working memory in heavy cannabis users: a functional magnetic resonance imaging study. Psychopharmacology (Berl) 176: 239-247.

Kim DI, Manoach DS, Mathalon DH, Turner JA, Mannell M, Brown GG et al (2009). Dysregulation of working memory and default-mode networks in schizophrenia using independent component analysis, an fBIRN and MCIC study. Hum Brain Mapp 30: 3795-3811.

Lane SD, Cherek DR, Lieving LM, Tcheremissine OV (2005). Marijuana effects on human forgetting functions. J Exp Anal Behav 83: 67-83.

Li YO, Adali T, Calhoun VD (2007). Estimating the number of independent components for functional magnetic resonance imaging data. Hum Brain Mapp 28: 1251-1266.

Mata I, Rodríguez-Sánchez JM, Pelayo-Terán JM, Pérez-Iglesias R, González-Blanch C, Ramírez-Bonilla M et al (2008). Cannabis abuse is associated with decision-making impairment among first-episode patients with schizophrenia-spectrum psychosis. Psychol Med 38: 1257-1266.

Matsuda LA (1997). Molecular aspects of cannabinoid receptors. Crit Rev Neurobiol 11: 143-166.
Mouslech Z, Valla V (2009). Endocannabinoid system: An overview of its potential in current medical practice. Neuro Endocrinol Lett 30: 153-179.

Pazos MR, Núñez E, Benito C, Tolón RM, Romero J (2005). Functional neuroanatomy of the endocannabinoid system. Pharmacol Biochem Behav 81: 239-247.

Pertwee RG (2008). The diverse CB1 and CB2 receptor pharmacology of three plant cannabinoids: delta9-tetrahydrocannabinol, cannabidiol and delta9-tetrahydrocannabivarin. $\mathrm{Br}$ J Pharmacol 153: 199-215.

Pope HG Jr, Gruber AJ, Hudson JI, Huestis MA, Yurgelun-Todd D (2001). Neuropsychological performance in long-term cannabis users. Arch Gen Psychiatry 58: 909-915.

Ruiz-Contreras AE, Carrillo-Sánchez K, Gómez-López N, VadilloOrtega F, Hernández-Morales S, Carnevale-Cantoni A et al (2013). Working memory performance in young adults is associated to the AATn polymorphism of the CNR1 gene. Behav Brain Res 236: 62-66.

Ruiz-Contreras AE, Carrillo-Sánchez K, Ortega-Mora I, BarreraTlapa MA, Romań-López TV, Rosas-Escobar CV et al (2014). Performance in working memory and attentional control is associated with the rs2180619 SNP in the CNR1 gene. Genes Brain Behav 13: 173-178.

Sambataro F, Blasi G, Fazio L, Caforio G, Taurisano P, Romano R et al (2010). Treatment with olanzapine is associated with modulation of the default mode network in patients with Schizophrenia. Neuropsychopharmacology 35: 904-912.

Schacht JP, Hutchison KE, Filbey FM (2012). Associations between cannabinoid receptor-1 (CNR1) variation and hippocampus and amygdala volumes in heavy cannabis users. Neuropsychopharmacology 37: 2368-2376.

Schmithorst VJ, Holland SK, Plante E (2006). Cognitive modules utilized for narrative comprehension in children: a functional magnetic resonance imaging study. Neuroimage 29: 254-266.

Schmithorst VJ, Holland SK, Plante E (2007). Object identification and lexical/semantic access in children: a functional magnetic resonance imaging study of word-picture matching. Hum Brain Mapp 28: 1060-1074.

Schnell T, Koethe D, Daumann J, Gouzoulis-Mayfrank E (2009). The role of cannabis in cognitive functioning of patients with schizophrenia. Psychopharmacology (Berl) 205: 45-52.

Seifert J, Ossege S, Emrich HM, Schneider U, Stuhrmann M (2007). No association of CNR1 gene variations with susceptibility to schizophrenia. Neurosci Lett 426: 29-33.

Sevy S, Burdick KE, Visweswaraiah H, Abdelmessih S, Lukin M, Yechiam E et al (2007). Iowa gambling task in schizophrenia: a review and new data in patients with schizophrenia and co-occurring cannabis use disorders. Schizophr Res 92: 74-84.

Solowij N, Stephens RS, Roffman RA, Babor T, Kadden R, Miller M et al (2002). Cognitive functioning of long-term heavy cannabis users seeking treatment. JAMA 287: 1123-1131.

Solowij N, Michie PT (2007). Cannabis and cognitive dysfunction: parallels with endophenotypes of schizophrenia? J Psychiatry Neurosci 32: 30-52.

Thompson-Schill SL, D’Esposito M, Aguirre GK, Farah MJ (1997). Role of left inferior prefrontal cortex in retrieval of semantic knowledge: a reevaluation. Proc Natl Acad Sci USA 94: 14792-14797.

van Winkel R, Genetic Risk and Outcome of Psychosis (GROUP) Investigators (2011b). Family-based analysis of genetic variation underlying psychosis-inducing effects of cannabis: sibling analysis and proband follow-up. Arch Gen Psychiatry 68: 148-157.

van Winkel R, van Beveren NJ, Simons C, Genetic Risk and Outcome of Psychosis (GROUP) Investigators (2011a). AKT1 moderation of cannabis-induced cognitive alterations in psychotic disorder. Neuropsychopharmacology 36: 2529-2537. 
Verdejo-García A, Beatriz Fagundo A, Cuenca A, Rodriguez J, Cuyás E, Langohr $\mathrm{K}$ et al (2013). COMT val158met and 5HTTLPR genetic polymorphisms moderate executive control in cannabis users. Neuropsychopharmacology 38: 1598-1606.

Wagner AD, Maril A, Bjork RA, Schacter DL (2001). Prefrontal contributions to executive control: fMRI evidence for functional distinctions within lateral Prefrontal cortex. Neuroimage 14: 1337-1347.
Weinberger DR, Egan MF, Bertolino A, Callicott JH, Mattay VS, Lipska BK et al (2001). Prefrontal neurons and the genetics of schizophrenia. Biol Psychiatry 50: 825-844.

Xu J, Zhang S, Calhoun VD, Monterosso J, Li CS, Worhunsky PD et al (2013). Task-related concurrent but opposite modulations of overlapping functional networks as revealed by spatial ICA. Neuroimage 79: 62-71. 